\title{
Functional LINC complex maintains epigenetic landscape and decondenses DNA in mature muscles
}

Daria Amiad Pavlov ${ }^{1}$, CP Unnikannan², Dana Lorber, Elizabeth Stoops, and Talila Volk*

Department of Molecular Genetics, Weizmann Institute of Science, Rehovot, Israel

1- Current address: Department of Physiology, University of Pennsylvania Perelman

School of Medicine

2- Current address: Bayer AG, Leverkusen 51368, Germany 


\begin{abstract}
The Linker of Nucleoskeleton and Cytoskeleton (LINC) complex transduces nuclear mechanical inputs suggested to control chromatin epigenetics. We analyzed the epigenetic landscape and genomic binding profile of HP1 and Polycomb transcription repressors, and of RNA-Pol II in Drosophila fully differentiated larval muscles lacking functional LINC complex. Our findings suggest a significant increase in chromatin repression promoted by enhanced binding of Polycomb and concomitant reduction of RNA-Pol II binding in the LINC mutant muscles. Consistently these mutants exhibited elevated levels of epigenetic repressive marks, tri-methylated $\mathrm{H} 3 \mathrm{~K} 9$ and $\mathrm{H} 3 \mathrm{~K} 27$, and reduced chromatin activation by $\mathrm{H} 3 \mathrm{~K} 9$ acetylation. These changes correlated with enhanced condensation of the DNA observed in the LINC mutant myonuclei. Importantly, we find larger repressive chromatin clusters marked by H3K27me3-GFP in live LINC mutant larval muscles. We suggest that the LINC complex is required for the stabilization of chromatin epigenetic landscape in non-dividing muscle fibers, possibly by inhibiting DNA condensation and restricting repressive cluster formation.
\end{abstract}




\section{Introduction}

The Linker of Nucleoskeleton and Cytoskeleton (LINC) complex, implicated in mechanical coupling between the cytoplasm and nucleoplasm, has been proposed to control chromatin organization and the epigenetic state of chromatin (Kirby and Lammerding, 2018; Lityagina and Dobreva, 2021; Poulet et al., 2017; Wagh et al., 2021). This complex consists of Nesprin proteins whose membrane-KASH domain is inserted into the outer nuclear membrane and their N-terminal associates with various cytoskeletal components. At the perinuclear space Nesprin tetramers bind covalently to SUN domain tetramers which further associate with the nuclear lamina and with chromatin (Chang et al., 2015; Razafsky and Hodzic, 2009; Sosa et al., 2012; Tapley and Starr, 2013; Wallrath et al., 2016). The functional contribution of the LINC complex for human health is displayed by numerous diseases associated with mutations in genes coding for components of the LINC complex, including Emery Dreifuss Muscular Dystrophy (EDMD), Arthrogryposis, Cerebral ataxia, Dilated Cardiomyopathy (DCM) and others (Cartwright and Karakesisoglou, 2014; Méjat and Misteli, 2010; Puckelwartz et al., 2009; Horn, 2014). Whereas the primary cause of LINC-associated diseases is not entirely clear, lack of functional LINC complex has been recently suggested to alter chromatin organization resulting in defects with gene transcription, and aberrant tissue function. For example, depletion of Nesprin-3 leads to nucleus collapse and loss of genome organization in adult rat cardiomyocytes (Heffler et al., 2019), LINC disruption in embryonic cardiomyocytes led to global chromatin and $\mathrm{H} 3 \mathrm{~K} 9 \mathrm{me} 3$ rearrangement (Seelbinder et al., 2021), plants deficient for components of the LINC complex, such as KASH (wifi) and SUN (sun1 sun4 sun5 triple mutant) show altered nuclear shape, increased distance of chromocenters from the nuclear periphery, altered heterochromatin organization and reactivation of transcriptionally silent repetitive sequences (Poulet et al., 2017). Likewise, mouse keratinocytes 
lacking SUN proteins exhibited precocious epidermal differentiation and loss of repressive chromatin H3K27me3 mark on differentiation-specific genes (Carley et al., 2021). In oligodendrocytes silencing of the Nesprin Syne1 gene resulted in aberrant histone marks deposition, chromatin reorganization and impaired gene transcription (Hernandez et al., 2016). Furthermore, in S. cerevisiae Mps3, a C-terminal SUN homologue is involved in the recruitment of heterochromatic sequences such as telomeric repeats to the NE, an essential process needed for spindle formation in the course of chromosome segregation (Ghosh et al., 2012). These studies implied that the LINC complex function associates with regulation of the epigenetic state of the chromatin, however the underlying mechanism is currently unclear. Moreover, most of these studies were performed either on cells in culture conditions, or on cells that were not fully differentiated. The contribution of the LINC complex to fully differentiated, non-dividing cells, where chromatin landscape has been established, is yet to be elucidated.

Here, we used Drosophila larval muscles as a model to investigate whether, and how the LINC complex impacts on chromatin activation or repression in mature, fully differentiated muscle fibers. Previously we showed that the LINC complex is required for correct larval muscle function, maintaining even distribution of nuclei along the entire muscle fiber (Elhanany-Tamir et al., 2012; Wang et al., 2015), and synchronization of myonuclear mechanical dynamics (Lorber et al., 2020). In addition, variable nuclear size, and elevated content of DNA due to precocious DNA endoreplication in the polyploid muscle nuclei was observed in the LINC mutant muscles (Wang et al., 2018). The higher DNA content and the variability in nuclear size suggested aberrant control of chromatin organization that could impact the epigenetic state of chromatin, linking LINCmediated mechanotransduction with chromatin organization and gene expression. To address this, we analyzed the genomic binding profile of the transcription repressors Polycomb and HP1, as 
well as that of RNA-Pol II, and quantified the levels of repressive epigenetic chromatin marks, H3K27me3 and H3K9me3 and active H3K9ac chromatin mark, in Drosophila larval muscles lacking functional LINC complex due to deletion of the single representative SUN domain gene klaroid (koi). We found that differentiated muscles lacking functional LINC complex exhibited increased epigenetic repression that correlated with enhanced genome binding of Polycomb and HP1, together with reduced binding of RNA-Pol II. The increased chromatin repression in the SUN/koi mutant muscle nuclei correlated with enhanced DNA condensation and with increased size of H3K27me3-marked chromatin clusters observed in live Drosophila larval muscles. These results suggest that lack of functional LINC complex alters the chromatin landscape in muscle nuclei, and promotes enhanced chromatin repression, through DNA condensation-dependent mechanism. Altogether these results imply that LINC-mediated mechanotransduction restrains gene repression in mature muscle fibers. 


\section{Results}

\section{Genome-wide Polycomb, HP1 and RNA-Pol II binding profiles reveal increased repression and reduced activation in $S U N /$ koi mutant muscle fibers.}

Although the LINC complex has been implicated in the regulation of epigenetic state, chromatin organization and overall gene-expression (Wagh et al., 2021), it is not clear whether and how LINC mediated mechanotransduction is involved in the downstream genome-wide chromatin repression and activation. Here, we investigated the contribution of the LINC complex to the epigenetic chromatin state by analyzing the phenotype of $S U N / k o i$ mutant muscles in Drosophila $3^{\text {rd }}$ instar larvae. Whereas the two Nesprin-like genes of Drosophila have additional functions in cells, due to non-KASH containing isoforms, SUN/koi, the only SUN representative in Drosophila does not appear to exhibit LINC-unrelated functions (Elhanany-Tamir et al., 2012; Titlow et al., 2020). We therefore investigated changes to genome-wide chromatin repression and activation states in the fully differentiated muscles of SUN/koi mutants in-vivo, using the Targeted DamID (TaDa) technique that allows tissue-specific profiling of DNA-binding proteins, in the intact living organism (Southall et al., 2013). Here we compared between DNA-binding profiles in SUN/koi mutated and control muscle fibers of two chromatin factors: (1) Polycomb, the H3K27me3 reader representative of the polycomb-group associated heterochromatin, (2) HP1, the $\mathrm{H} 3 \mathrm{~K} 9 \mathrm{me} 3$ reader representative of the HP1-associated heterochromatin, and in addition, the profile of RNA-Pol II subunit representative of actively transcribed euchromatin. Polycomb, HP1, and RNA-pol II fused to the DNA adenine methyltransferase (Dam) were driven to Drosophila larval muscles using Mef2-GAL4 driver, in a temporally controlled manner (by combined expression of the temperature sensitive inhibitor $\mathrm{Gal} 80^{\mathrm{ts}}$ ), inducing expression of the Dam-fusion proteins only at the $3^{\text {rd }}$ instar larval stage, when muscles are mature and fully differentiated. The 
experiment included N=3 independent replicates of Dam-Polycomb, Dam-HP1, Dam-RNA Pol II, and Dam only (as reference), for control and for SUN/koi nutant genotypes, with $\mathrm{n}=25$ larvae in each group. Following 10 hours of Dam expression, larvae were dissected, DNA was extracted and DpnI digested at the Dam methylated GATC sites. Further amplification, processing, and bioinformatics analysis were performed as described (Marshall and Brand, 2015).

To compare gene binding profiles between SUN/koi and control muscle fibers, we performed a PCA regression analysis on Dam-Polycomb, Dam-HP1, and Dam-Pol II, normalized to Dam binding alone (Figure 1A). The cut-off criteria for significant change in gene binding between $S U N / k o i$ and control was set to false discovery rate (FDR) $<0.05$, z-score $>1.96$, and GATC sites > 1. Gray dots in Figure 1A represent genes with significantly altered binding, such that genes below the regression line represent increased binding in the $S U N /$ koi mutant, and genes above the regression line represent decreased binding in $S U N / k o i$, compared to control. Overall, we detected significantly altered binding to 148 genes in the Polycomb group, 173 genes in the HP1 group and 206 genes in the RNA-Pol II group. Note the robust increase in Polycomb binding observed in the SUN/koi mutant, where 143 out of 148 significant genes showed enhanced Polycomb occupancy. Figure 1B demonstrates Dam-Polycomb, Dam-HP1, and Dam-Pol II occupancy in the alphaTub84B (left), Nup62 (middle), and Act57B (right) genes. Interestingly, each of these genes showed altered occupancy in SUN/koi mutant muscles for only one chromatin factor (red boxes) with increased Polycomb binding to alphaTub84B, increased HP1 binding to Nup62, and decreased RNA-Pol II binding to the Act57B gene. 
A
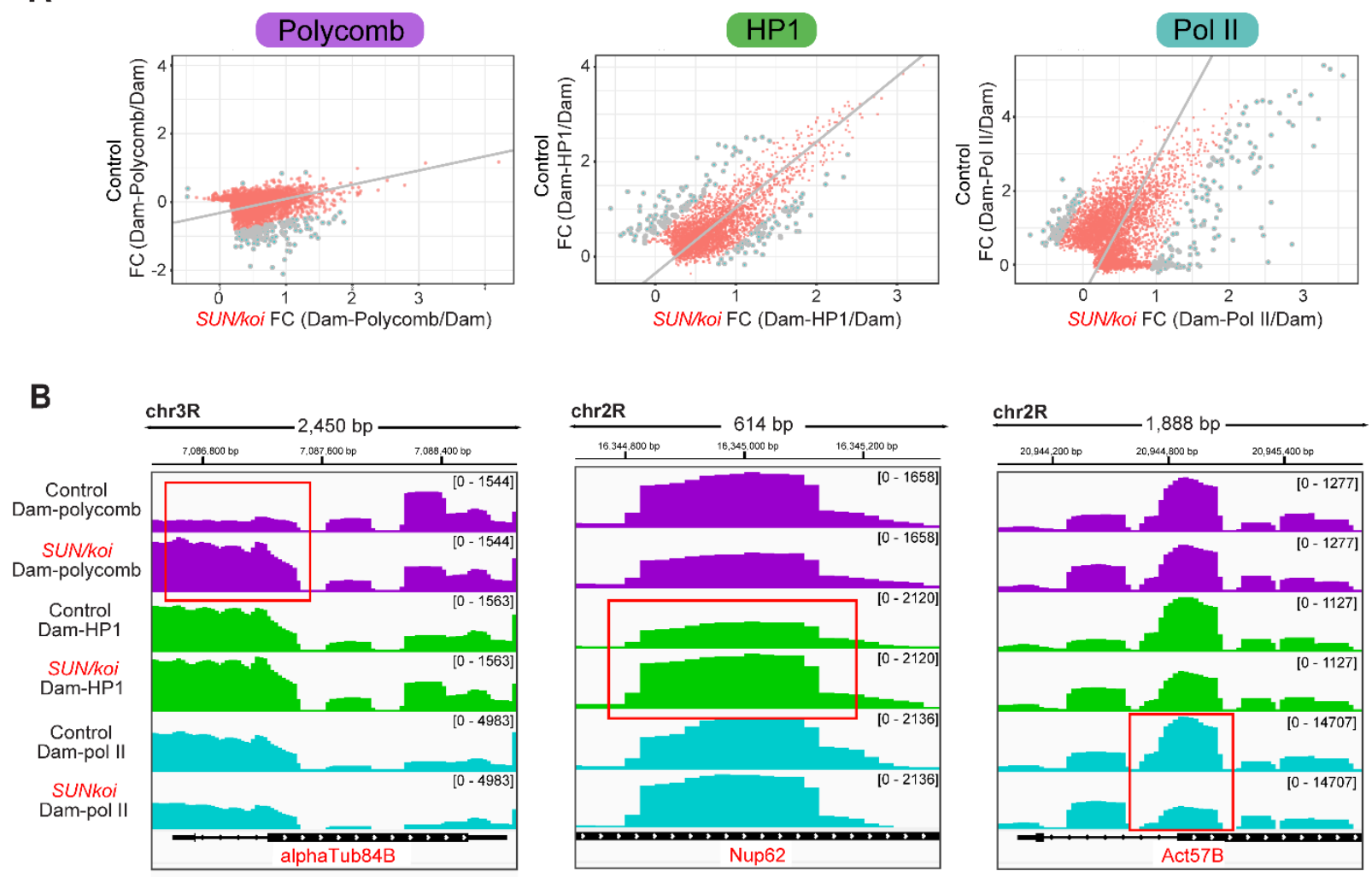

Figure 1. Increased Polycomb and altered HP1 and RNA-Pol II occupancies in LINC mutated muscle fibers. (A) PCA regression on Dam-Polycomb (left), Dam-HP1 (middle), DamPol II (right) gene occupancy, normalized to Dam alone, in control versus SUN/koi mutated muscle fibers. Gray dots represent genes with significantly altered binding profile when cut-off criteria are set to FDR $<0.05$, Z-score >1.96, GATC sites $>1$. (B) Dam-Polycomb, Dam-HP1, and Dam-Pol II, occupancy, normalized to Dam alone, for the alphaTub84B (left), Nup62 (middle), and Act57B (right) genes. Each gene presents altered occupancy in only one chromatin factor upon SUN/koi mutation (highlighted with red box).

We further paralleled genome wide changes in Polycomb, HP1 and RNA-Pol II occupancy in the $S U N / k o i$ mutant muscles by comparing the significantly altered genes in each group. Figure 2A depicts a heatmap of (SUN/koi versus control) fold change occupancy, for each chromatin factor, such that red indicates increased occupancy and blue indicates decreased occupancy as observed in SUN/koi mutant muscles. Non-significant genes are labeled in gray. Gene clustering was defined by k-means, and the number of clusters was estimated with Gap statistics. Overall, we observe minimal overlap between the group of genes that showed altered binding to the three 
factors, namely, Polycomb, HP1, and RNA-Pol II, as the majority of genes colored in each group are non-significant (gray) in the others. Cluster 1 in Figure 2A represents mostly genes that showed increased Polycomb occupancy. A subgroup of these genes shared also increased HP1 binding, and therefore are predicted to undergo significant repression. Likewise, another subgroup within cluster 1 also shared decreased RNA-Pol II binding, predicting an overall reduced expression for these genes. Cluster 2 represents a distinct group of genes that showed decreased RNA-Pol II binding, thus expected to be downregulated. Cluster 3 includes genes that exhibited mostly increased RNA-Pol II binding, and therefore expected to be upregulated. Interestingly HP1 did not show a clear tendency of altered binding. Similar non-overlap in altered genes among the three chromatin binding groups is shown in Figure 2B which lists specific, significantly altered genes of interest, and their corresponding fold change value in the SUN/koi mutant. Muscle specific genes are mostly down-regulated through decreased RNA-Pol II or increased Polycomb binding. Interestingly we also observed decreased binding of RNA-Pol II and increased binding of HP1 to a group of genes involved in nuclear transport. A group of transcriptional regulators showed increased Polycomb binding and decreased RNA-Pol II binding. Figure 2C demonstrates a gene ontology (GO) enrichment analysis (Zhou et al., 2019) on the three clusters of genes identified in Figure 2A. Cluster 1, mostly associated with increased Polycomb binding, is enriched for TOR signaling pathway (R-DME-165159 and GO:0032006) and negative regulation of catalytic activity (GO:0043086), indicative of repressing major growth related signaling pathways in the SUN/koi mutant muscle fibers. Cluster 2, mostly associated with decreased RNA-Pol II binding, is enriched for muscle specific GO terms (GO:0016460, GO:0043292), indicative of reducing transcription of muscle specific genes in the SUN/koi mutant. 
Taken together our genome-wide analysis indicates that defective LINC complex associates with increased chromatin repression, mostly through increased Polycomb binding, and decreased transcriptional activation through reduced RNA-Pol II binding. Interestingly each chromatin factor group is involved in the regulation of a distinct group of genes with only minimal overlap in genes controlled by Polycomb, HP1, and RNA-Pol II. This is consistent with genome binding maps illustrating no overlap between Polycomb and HP1 repressive chromatin (Filion et al., 2010; De Wit et al., 2007).

A

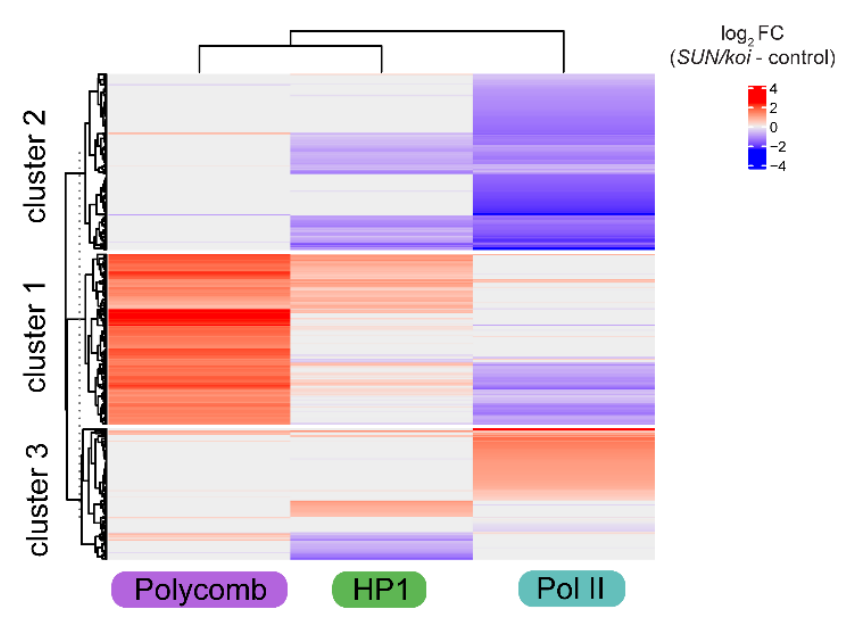

C

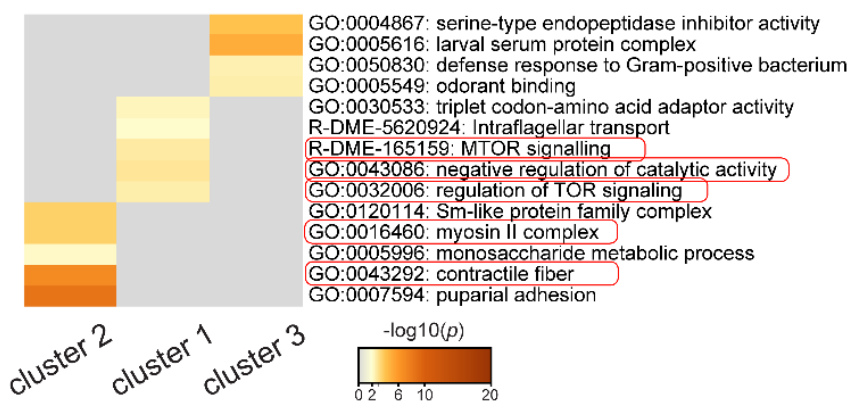

B

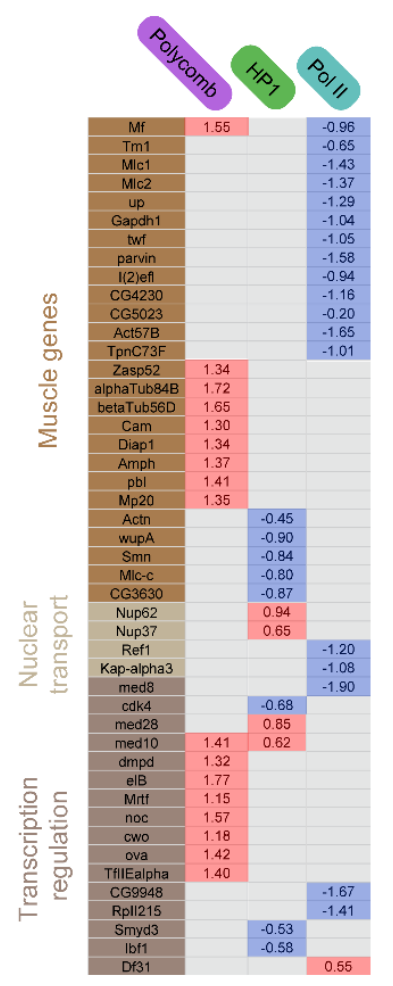

Figure 2. Polycomb, HP1, and RNA-Pol II binding regulate distinct group of genes in the LINC mutated muscle fibers, with overall increased Polycomb repression and decreased RNA-Pol II activation. (A) The difference in SUN/koi and control fold change occupancy represented as heatmap for significantly altered genes in the Polycomb, HP1, and RNA-Pol II groups. Non-significant genes are labeled in gray. K-means gene clustering reveals downregulated genes in cluster 1 (increased Polycomb binding), and cluster 2 (decreased RNA-Pol II 
binding), and mostly up-regulated genes in cluster 3 (increased RNA-Pol II). (B) The difference in $S U N / k o i$ and control fold change occupancy for specific genes of interest. (C) GO enrichment analysis (heatmap of $p$-values) on the three clusters of genes identified in (A).

\section{Perturbed LINC complex associates with increased epigenetic chromatin repression, and reduced epigenetic chromatin activation in muscle nuclei}

The changes in Polycomb and RNA-Pol II genome binding led us to address possible changes in the meso-scale epigenetic landscape in $S U N / k o i$ mutated muscle fibers of $3^{\text {rd }}$ instar larvae, using antibody staining for three epigenetic modifications, namely H3K9ac, H3K27me3, and $\mathrm{H} 3 \mathrm{~K} 9 \mathrm{me} 3$. Figure $3 \mathrm{~A}$ shows representative muscle nuclei labeled with the repressive chromatin modifications H3K27me3 (purple), and H3K9me3 (green), with overall increased signal of both marks, in the SUN/koi mutated muscle nuclei. Figure 3B shows quantification for the mean nuclear fluorescence intensity with $43 \%$ increase in $\mathrm{H} 3 \mathrm{~K} 27 \mathrm{me} 3(\mathrm{p}<0.05)$, and $82 \%$ increase in $\mathrm{H} 3 \mathrm{~K} 9 \mathrm{me} 3$ ( $\mathrm{p}<0.01)$. The overall increased intensity of repressive chromatin marks is in agreement with the increased Polycomb binding observed with the targeted DamID. As previously reported, the SUN/koi mutant displays smaller and variable myonuclear size and increased DNA ploidy (Wang et al., 2018). We therefore asked whether the observed epigenetic changes correlate with the nuclear size. Figure 3C shows the mean H3K27me3, and H3K9me3 fluorescence intensity, for each nucleus, as function of the nuclear volume (log10 scale). Interestingly, both of the repressive marks displayed increased repression with smaller nuclear volumes. We performed linear mixed model analysis on the dependence of mean H3K27me3 intensity (left), and mean H3K9me3 intensity (right) on nuclear volume in the $S U N /$ koi group, compared to control (red and black dots, respectively). We first compared fitted mixed models for all pulled nuclear volumes and found significant differences between the $S U N / k o i$ and control 
groups, for $\mathrm{H} 3 \mathrm{~K} 27 \mathrm{me} 3$ and $\mathrm{H} 3 \mathrm{~K} 9 \mathrm{me} 3$ repressive marks $(\mathrm{p}<0.01)$. To assess the contribution of the smaller nuclei, which present only in the SUN/koi groups, we then fitted mixed models only for the larger nuclei, with overlapping volumes between the SUN/koi and control groups. The larger, overlapping volume nuclei showed no significant difference between the $S U N / k o i$ and control models, for both repressive marks $(p>0.2)$, suggesting that the smaller nuclei in the SUN/koi groups are the major contributors to the increased $\mathrm{H} 3 \mathrm{~K} 27 \mathrm{me} 3$ and $\mathrm{H} 3 \mathrm{~K} 9 \mathrm{me} 3$ repression. In agreement with the reduced RNA-Pol II binding to muscle specific genes, we identify a decrease in $\mathrm{H} 3 \mathrm{~K} 9 \mathrm{ac}$ chromatin modification, which marks the active promotors, in the SUN/koi mutant muscle fibers (Figure 4A). Figure 4B quantifies the mean nuclear H3K9ac intensity with $37 \%$ reduction in the $S U N / k o i$ mutant $(\mathrm{p}<0.01)$. In contrast to the repressive marks, the active H3K9ac mark showed no significant correlation with nuclear volume (Figure 4C). Overall, this data further supports increased repressive and reduced active landscape in the SUN/koi mutated muscle fibers. Increased repression is further correlated with smaller nuclear volume pointing to the link between chromatin condensation and epigenetic repression in LINC perturbed muscles. 
bioRxiv preprint doi: https://doi.org/10.1101/2022.02.09.479725; this version posted February 10,2022 . The copyright holder for this preprint (which was not certified by peer review) is the author/funder, who has granted bioRxiv a license to display the preprint in perpetuity. It is made available under aCC-BY-NC-ND 4.0 International license.

A

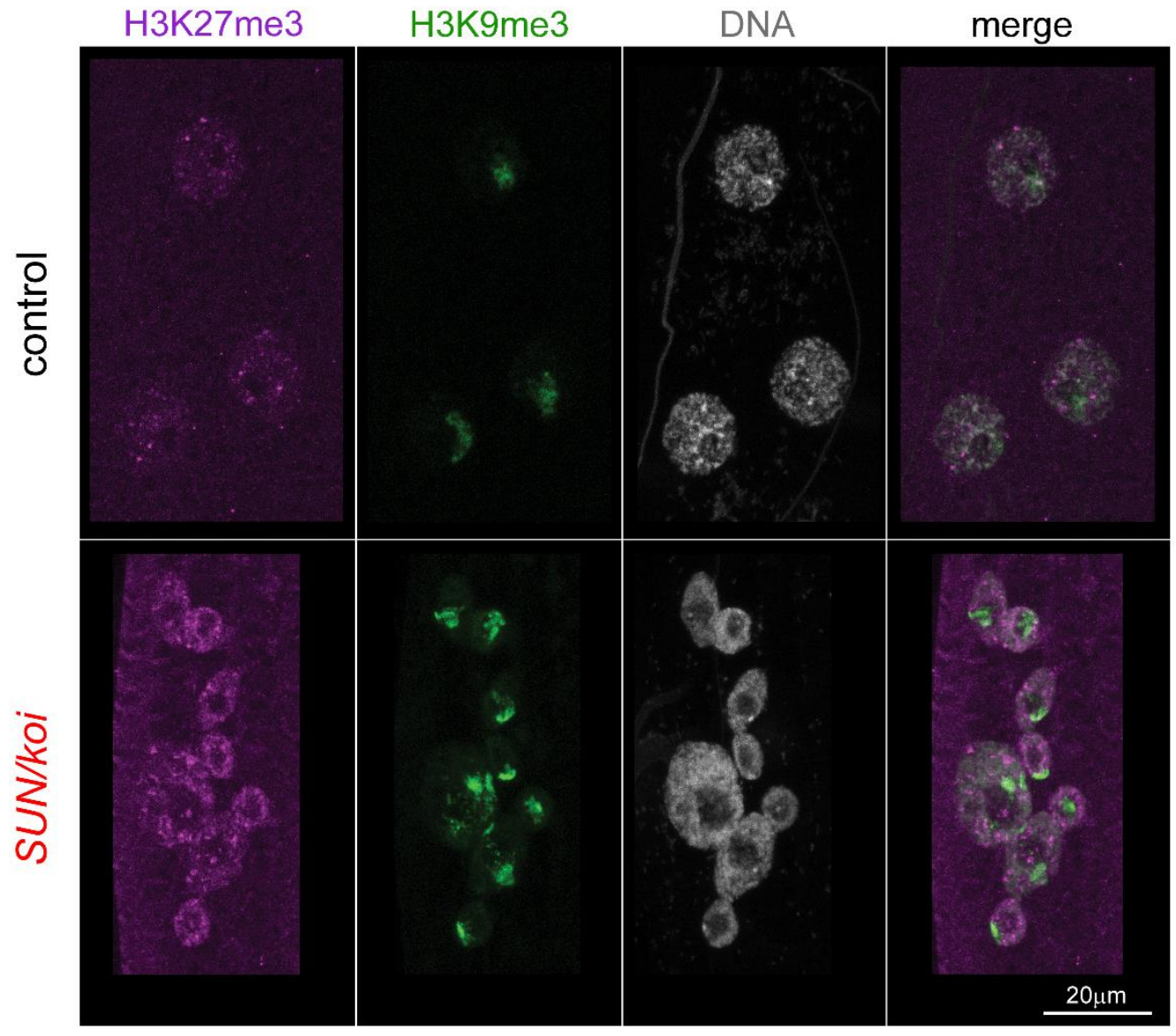

B
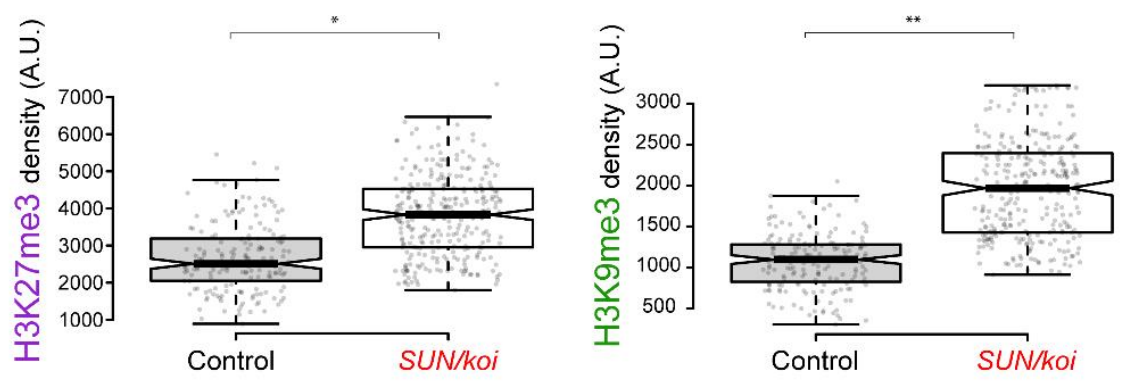

C
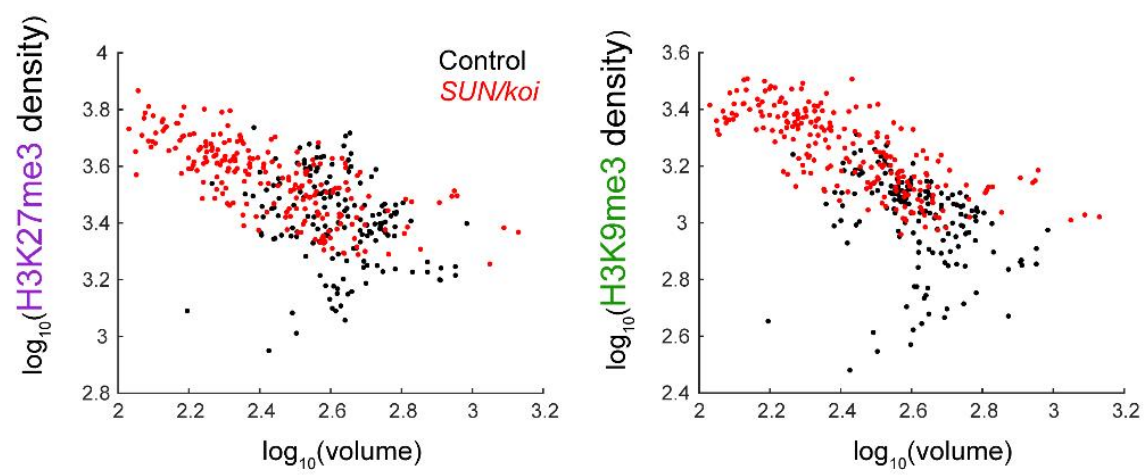
Figure 3. Increased repressive H3K27me3 and H3K9me3 chromatin density, inversely correlated with nuclear volume, in SUN/koi mutated muscle fibers. (A) Muscle nuclei labeled with H3K27me3 (purple), H3K9me3 (green), and Hoechst for total DNA (gray). (B) Quantification of mean nuclear fluorescence intensity shows increased H3K27me3 and H3K9me3 in $S U N / k o i$ mutated muscle fibers. (C) Mean nuclear fluorescence intensity is plotted against the corresponding nuclear volume ( $\log 10$ scale) for each epigenetic mark. Significant difference in the linear mixed model fit between the $S U N / k o i$ and control groups, for both repressive marks $(\mathrm{p}<0.01)$. Similar analysis comparing only the larger nuclei (overlapping in $S U N / k o i$ and control groups) shows no significant difference between the fits, suggesting that mostly the smaller nuclei in the SUN/koi mutant contribute to the increased $\mathrm{H} 3 \mathrm{~K} 27 \mathrm{me} 3$ and $\mathrm{H} 3 \mathrm{~K} 9 \mathrm{me} 3$ repression. $\mathrm{N}=5$ larvae, $\mathrm{n}=177$ nuclei in control, $\mathrm{N}=5$ larvae, $\mathrm{n}=295$ nuclei in $S U N / k o i$. For statistical significance ${ }^{*} \mathrm{p}<0.05,{ }^{* *} \mathrm{p}<0.01$.

A

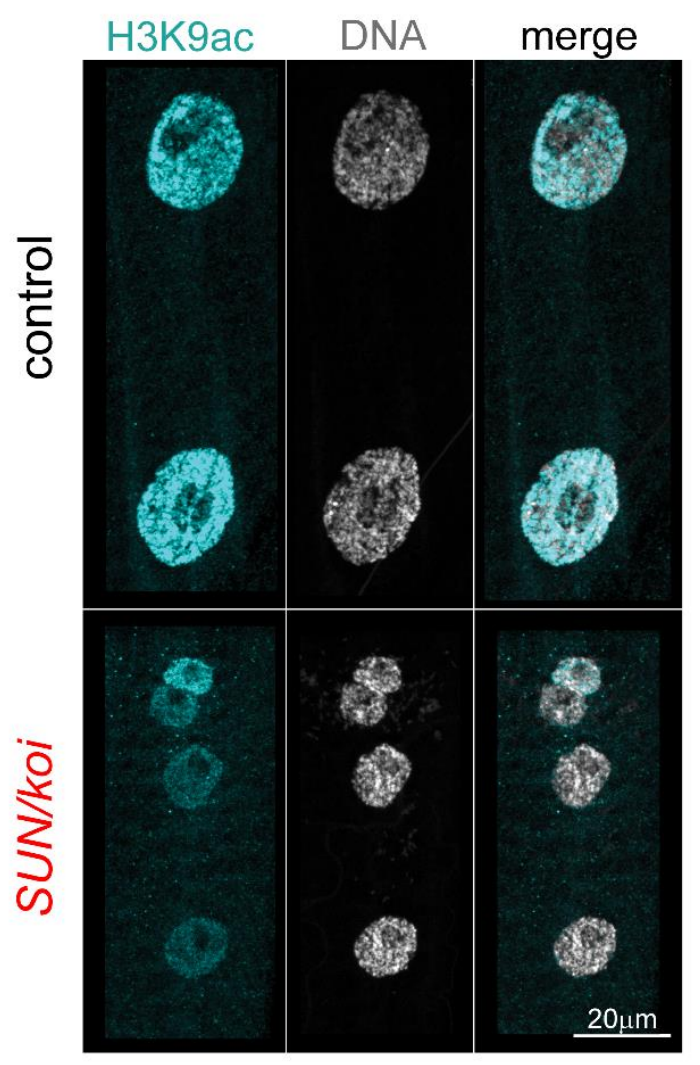

B

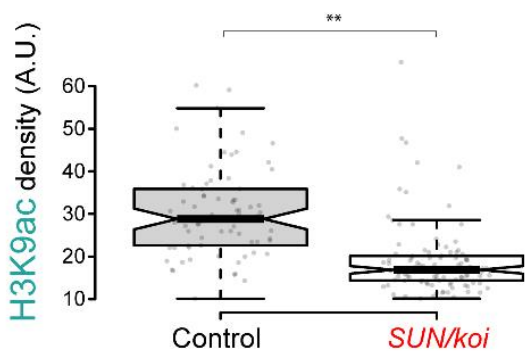

C

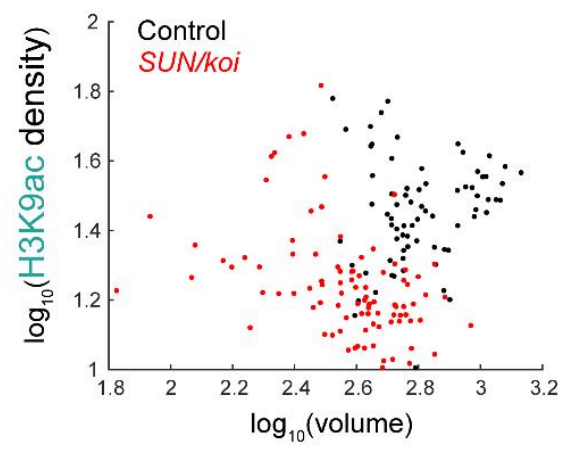

Figure 4. Reduced active H3K9ac chromatin density in SUN/koi mutated muscle fibers, independent of nuclear volume. (A) Muscle nuclei labeled with H3K9ac (cyan) and Hoechst for total DNA (gray). (B) Quantification of mean nuclear fluorescence intensity shows decreased H3K9ac in SUN/koi mutated muscle fibers. (C) Mean nuclear H3K9ac intensity is plotted against the corresponding nuclear volume $(\log 10$ scale) and shows no significant correlation with nuclear volume between the control and SUN/koi groups (left). N=5 larvae, $\mathrm{n}=73$ in control, $\mathrm{N}=4$ larvae, $\mathrm{n}=103$ nuclei in $S U N / k o i . * * \mathrm{p}<0.01$. 


\section{Increased DNA condensation in SUN/koi mutant muscle nuclei}

Drosophila muscle nuclei are polyploid, where the chromatin volume scales linearly with nuclear volume to maintain constant chromatin condensation, in the wild-type nuclei (AmiadPavlov et al., 2021). The inverse correlation of chromatin repression with nuclear volume, observed in the SUN/koi mutant muscles, suggested that increased chromatin condensation might contribute to the excessive chromatin repression (Schuettengruber et al., 2017). To address this, we compared the global (nuclear scale) and local ( $\sim 3 \mathrm{Mbp}$ genomic regions) DNA condensation in SUN/koi and control myonuclei. Figure 5A shows representative images of control and SUN/koi mutant muscle nuclei, stained with Hoechst, and used to compare the global DNA condensation between the groups. Note that $S U N / k o i$ mutant muscle nuclei are often smaller than control and on average contain more DNA as a result of DNA endoreplication (Wang et al., 2018). Figure 5B shows a plot of the total DNA intensity as a function of nuclear volume (log10 scale) for SUN/koi and control groups (red and black dots, respectively). A linear mixed model analysis confirms a significant difference between the $S U N /$ koi and control fits $(\mathrm{p}<0.01)$ with a leftward-shift in the $S U N / k o i$ total DNA - volume relationship, indicating that for each $S U N / k o i$ nucleus the DNA is condensed in a smaller nuclear volume, compared to control. Such condensation might underly the basis for the enhanced repression observed in the SUN/koi mutant muscle nuclei. As the LINC complex transmits mechanical signals from the cytoskeleton into the nucleus, we asked if perturbation of upstream mechanical force applied on the nuclear envelope will have similar effect on nuclear size and DNA condensation. To this end we perturbed sarcomeric forces applied on the nuclear envelope by muscle specific knockdown of D-titin (sls-RNAi), as well as forces resulting from the association of the microtubule system with the nuclear envelope by muscle-specific overexpression of spastin, a microtubule severing protein. Both approaches resulted in increased 
nuclear volume but in contrast to the LINC mutant muscle nuclei, the linear scaling between total DNA and nuclear volume was preserved (Figure S1), indicative of maintained global chromatin condensation. We therefore concluded that altered scaling between DNA content and nuclear volume observed in the $S U N /$ koi mutant muscles correlates with the epigenetic changes observed in these mutants.

We then assessed the effect of SUN/koi mutation on local DNA condensation by threecolor Oligopaint FISH, developed by the Joyce lab (Rosin et al., 2018), targeting distinct 3-4Mb domains on the Drosophila 2L chromosome. The probes included oligos reactive with DNA sequences near the centromere (green), at the middle (red), and close to the telomere (purple) of chromosome 2 left arm (Figure 5C). We quantified probes signal shape parameters, as well as its distance to nuclear center, and found a significant reduction in the surface area of the near centromeric probe signal (green), in the $S U N / k o i$ mutant muscles ( $\mathrm{p}<0.05)$, while middle (red) and telomeric (purple) signals showed no significant change in the surface area between the groups (Figure 5D). The distance of the centromeric signal to the nucleus center (normalized to nuclear long axis to account for smaller nuclei in the mutant), was also smaller in the SUN/koi mutant compared to control, while no significant changes were observed in the other signals (Figure 5E). These findings suggest that the global DNA condensation associated with the $S U N /$ koi mutation is translated heterogeneously into local DNA compaction and spatial rearrangement. 
A

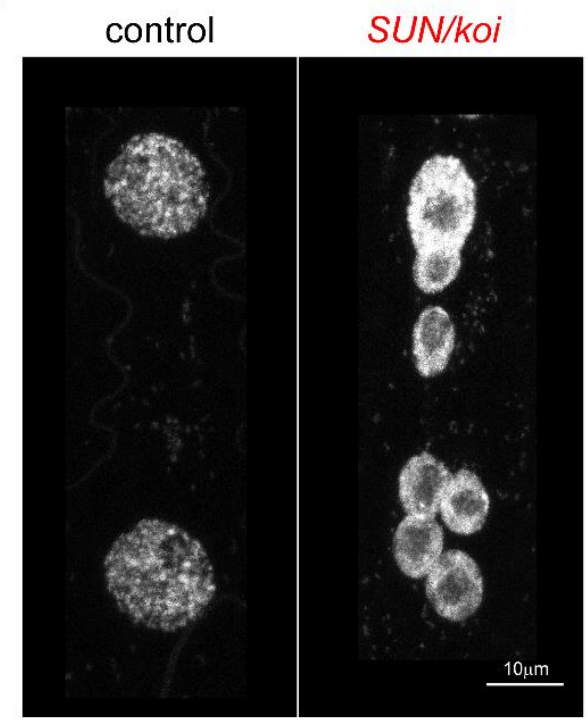

C

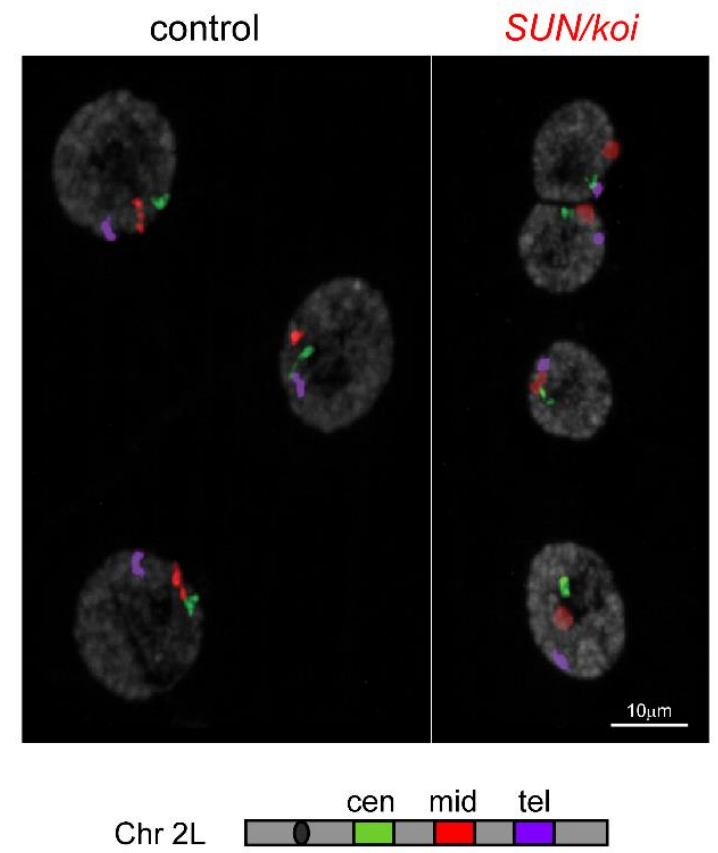

B

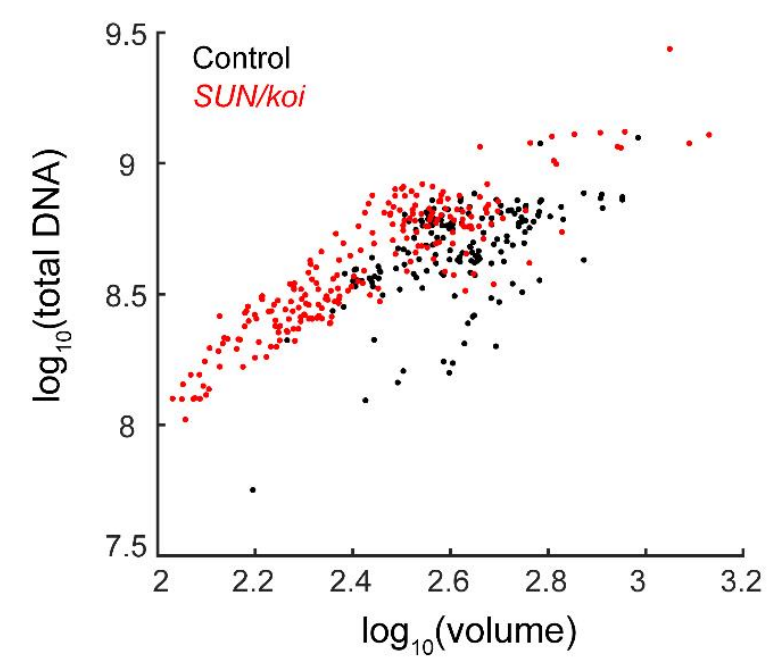

D

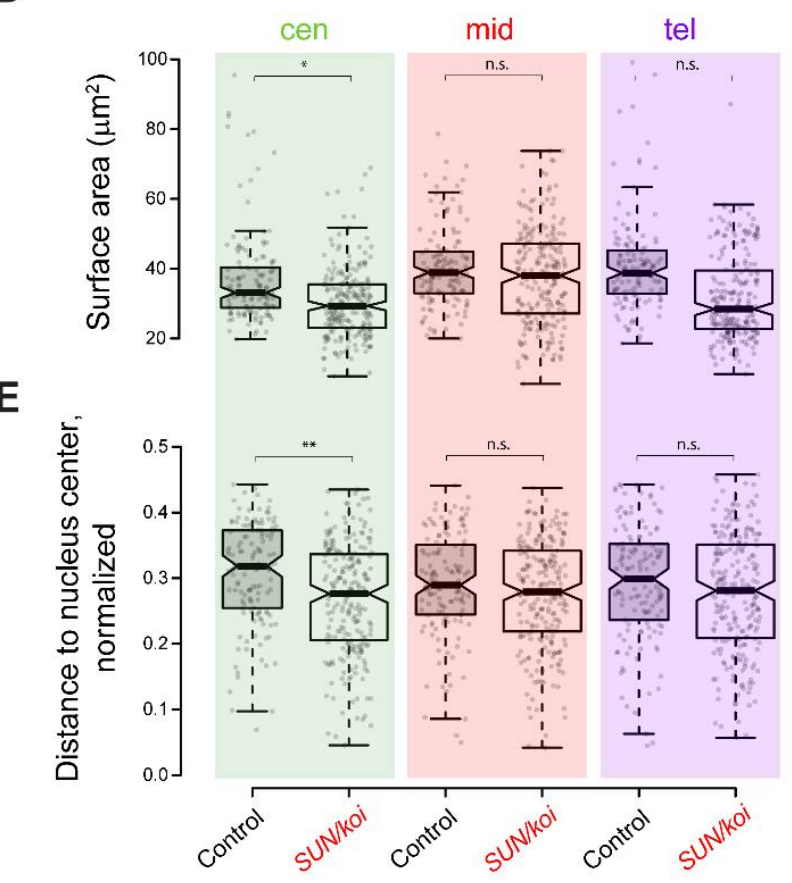

Figure 5. Global DNA condensation in the $S U N / k o i$ mutant muscle nuclei is reflected heterogeneously in local chromatin regions. (A) Control and SUN/koi muscle nuclei labeled with Hoechst. (B) Total DNA intensity plotted versus nuclear volume (log 10 scale) for $S U N / k o i$ (red) and control (black). Linear mixed model analysis confirms significant difference between the SUN/koi and control fits $(\mathrm{p}<0.05)$, with left-ward shift in the total DNA - nuclear volume relationship for the $S U N /$ koi group. $\mathrm{N}=5$ larvae, $\mathrm{n}=177$ nuclei in control, $\mathrm{N}=5$ larvae, $\mathrm{n}=295$ nuclei in SUN/koi. (C) Control and SUN/koi muscle nuclei simultaneously labeled with 3-color DNAFISH Oligopaint probes at near centromeric (green), near middle (red), and near telomeric (purple) 
regions of Drosophila chromosome 2L. (D+E) Quantification of probe signal surface-area, and distance to nucleus center (normalized to nuclear length). $\mathrm{N}=4$ larvae, $\mathrm{n}=133$ nuclei in control, $\mathrm{N}=5$ larvae, $\mathrm{n}=228$ nuclei in SUN/koi. For statistical significance* $\mathrm{p}<0.05,{ }^{*} \mathrm{p}<0.01$.

\section{Live H3K27me3 3D imaging reveals increased spreading of the repressive chromatin mark in $S U N / k o i$ mutated muscle fibers}

Our previous work indicated peripheral chromatin organization in nuclei of mature muscle fibers, which is sensitive to lamin A/C levels, when imaged live, in-vivo (Amiad-Pavlov et al., 2021). To assess the meso-scale chromatin organization in $S U N /$ koi mutated muscle we imaged live larval myonuclei co-labeled with H2B-mRFP and Klar-GFP. However, in the SUN/koi mutant, the Klar-GFP did not localize to the nuclear envelope (due to the lack of $S U N / k o i$ ). The overall peripheral chromatin organization was still noticeable in the live $S U N / k o i$ mutant from the H2BmRFP channel alone (Figure S2). To investigate the nature of the overall increase in H3K27me3 density and increased Polycomb binding profile in the $S U N /$ koi mutants, we performed live 3D imaging of larval muscles utilizing the H3K27me3-GFP mintbody (Tjalsma et al., 2021). Representative 3D spatial distribution of H3K27me3-GFP in control and SUN/koi mutated nuclei is shown in Movie S1 and Movie S2, respectively. Figure 6A shows middle confocal sections of representative control (left) and SUN/koi mutant (right) muscle nuclei labeled with H3K27meGFP mintbody. The strong H3K27me3 signal appears punctuated within the nucleus, with weaker nuclear and cytoplasmic background. Compared to control distribution, the H3K27me3 puncta in the $S U N / k o i$ mutant nuclei appeared more spread-out throughout the chromatin, resulting in larger repressive H3K27me3 hubs. We quantified the spread of the puncta by measuring the volume (Figure 6B) and the number (Figure 6C) of strong H3K27me3 puncta in each group. The SUN/koi puncta volume is on average $149 \%$ higher than control $(\mathrm{p}<0.01)$ with no significant change in the 
number of the puncta between the groups. Thus, the live 3D distribution of H3K27me 3 puncta demonstrates increased spreading of this repressive chromatin mark in the SUN/koi mutated muscle nuclei. Taken together with the increased Polycomb binding and DNA condensation, our findings suggest that $S U N /$ koi disruption induces increased repression by DNA condensation and spread of repressive $\mathrm{H} 3 \mathrm{~K} 27 \mathrm{me} 3$ chromatin modifications to nearby chromatin regions.

A

Control
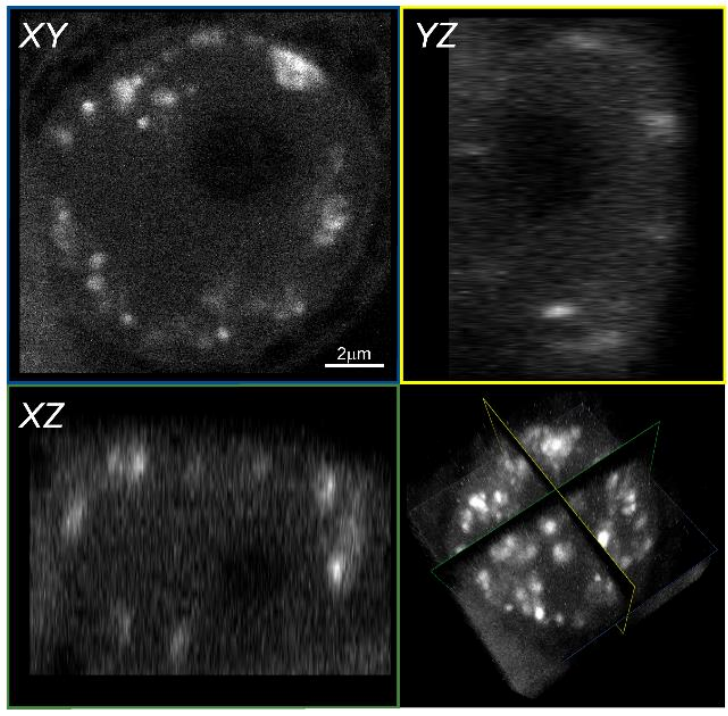

B
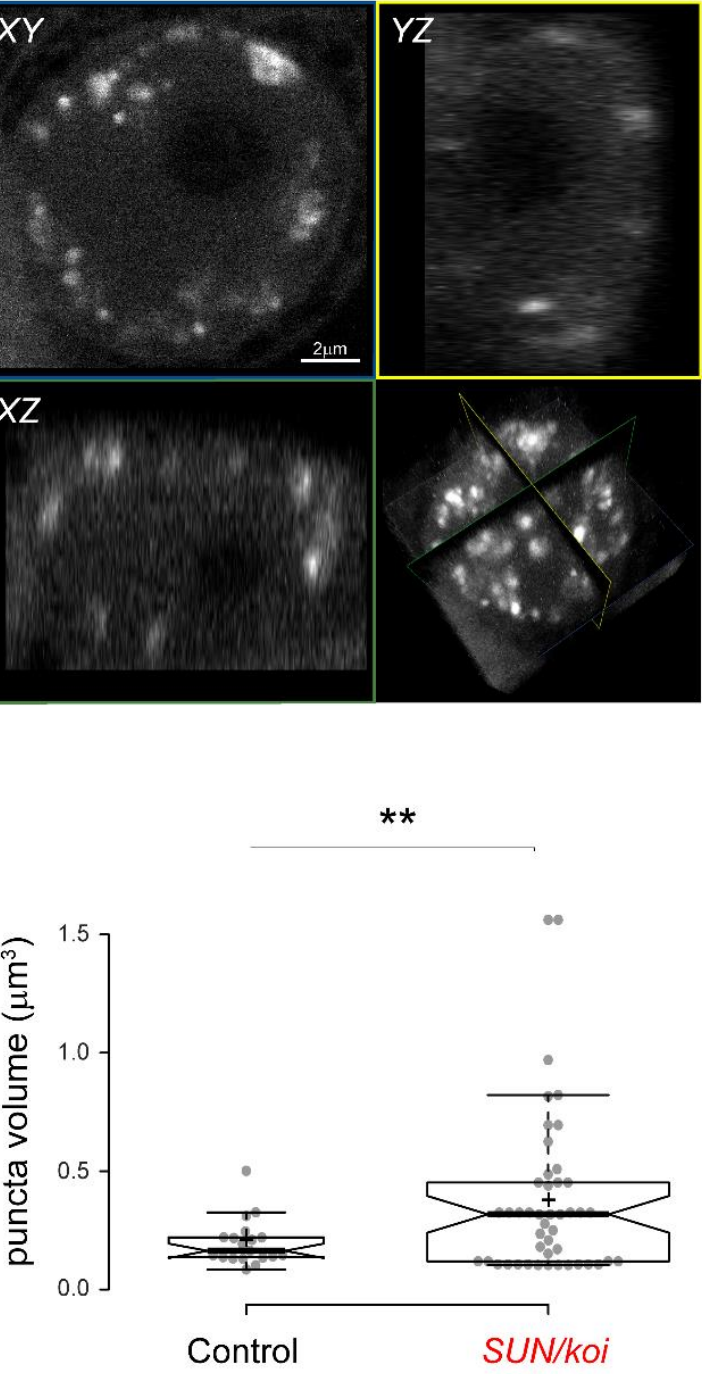

C

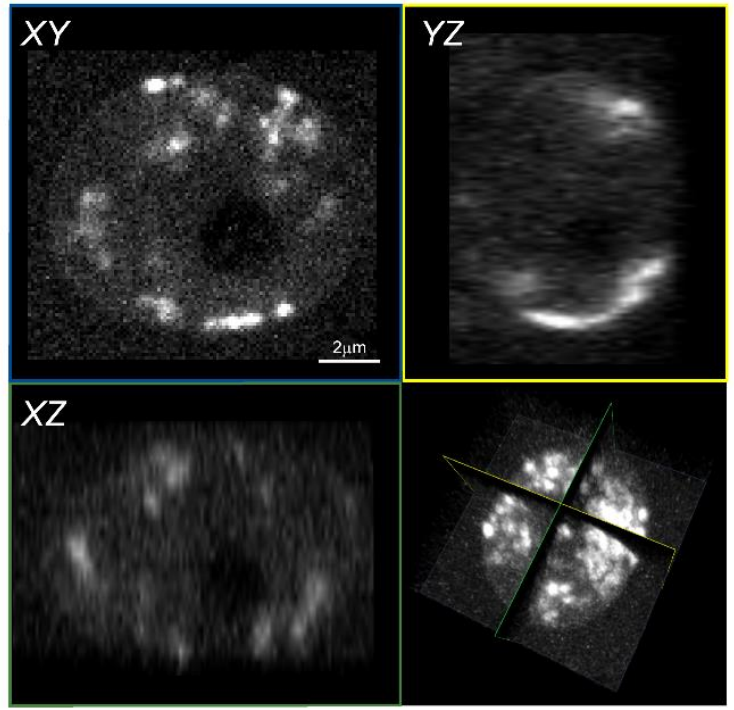

H3K27me3-GFP

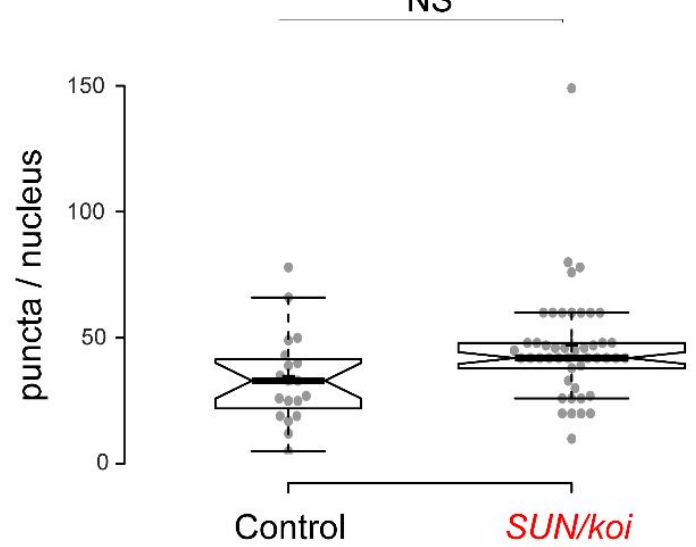

Figure 6: Live 3D imaging of H3K27me3 mintbody distribution indicates increased spread of the repressive mark in $S U N / k o i$ mutant muscle nuclei. (A) Middle confocal planes of control (left) and $S U N / k o i$ (right) muscle nuclei show punctuated repressive regions throughout the nucleus. Increased mean puncta volume (B) and unchanged number of puncta per nucleus (C) in 
bioRxiv preprint doi: https://doi.org/10.1101/2022.02.09.479725; this version posted February 10, 2022. The copyright holder for this preprint (which was not certified by peer review) is the author/funder, who has granted bioRxiv a license to display the preprint in perpetuity. It is made available under aCC-BY-NC-ND 4.0 International license.

the $S U N /$ koi mutant group. $\mathrm{N}=4$ larvae, $\mathrm{n}=19$ nuclei in control, $\mathrm{N}=4$ larvae, $\mathrm{n}=46$ nuclei in $S U N /$ koi $(* * \mathrm{p}<0.01)$. 


\section{Discussion}

The LINC complex has been implicated in the regulation of chromatin structure; however, the underlying mechanism is yet to be elucidated. Here we addressed the contribution of the LINC complex to 1) the chromatin-binding profile of specific transcription regulators 2) the chromatin epigenetic landscape and 3) the condensation of DNA, in differentiated Drosophila muscle fibers. We demonstrate altered genome-wide binding profiles of Polycomb, HP1, and RNA-Pol II in SUN/koi mutant muscles, with specific increased binding of the transcription silencer Polycomb, and decreased binding of RNA-Pol II, to distinct groups of genes. This altered binding profile predicts enhanced transcriptional repression in the $S U N /$ koi mutant muscle fibers, which is further supported by increased levels of the repressive tri-methylated H3K27 and H3K9, as well as decreased levels of the active H3K9ac chromatin marks. Further analysis indicated that these changes correlated with LINC mutant nuclei displaying higher DNA density, leading to local DNA condensation. Remarkably, live imaging of tri-methylated H3K27 marked chromatin reveals larger repressive chromatin hubs in $S U N /$ koi mutant muscles. Taken together these results demonstrate that the LINC complex is required for inhibition of excessive chromatin epigenetic repression, assumed to be required for stabilization of chromatin structure and robust transcriptional regulation in fully differentiated muscle fibers.

Polycomb genome-wide binding profile showed a clearly directed trend with increased binding to a group of 143 genes (out of total 148 genes with altered binding) observed in the SUN/koi mutant muscles, which implies a significantly enhanced transcription repression. Interestingly, among these genes (cluster 1) we identified enrichment for two GO terms functionally associated with Tor signaling, a major signaling pathway regulating muscle growth. Enhanced transcriptional repression of Tor signaling in the SUN/koi mutants is consistent with the 
thinner muscle phenotype observed in these muscles (Elhanany-Tamir et al., 2012; Wang et al., 2018). Furthermore, since Polycomb binds specifically to tri-methylated H3K27-marked chromatin, the enhanced Polycomb binding is also consistent with an increased H3K27me3 fluorescent labeling observed in the SUN/koi mutant muscles. At this stage we cannot determine whether increased polycomb binding leads to increased H3K27me3 or vice versa, since Polycomb was demonstrated to self-aggregate with chromatin due to phase separation (Tatavosian et al., 2019), but also, to further promote tri-methylation of H3K27 (Schuettengruber et al., 2017). Our observation that in muscles of live $S U N /$ koi mutant larvae the H3K27me3-positive puncta grew in size is consistent with increased self-aggregation of pre-existing $\mathrm{H} 3 \mathrm{~K} 27$ me3-associated chromatin clusters.

Polycomb is a member of polycomb repressive complexes (PRCs) and has been extensively studied in development and differentiating cells. PRC binding to epigenetically modified chromatin leads to transcriptionally poised state, which then prevents unscheduled differentiation (Schuettengruber et al., 2017). Therefore, enhanced binding of Polycomb to chromatin might inhibit transcriptionally regulated developmental transitions in the larval muscle fibers. The idea that such transitions might be regulated by mechanical signals, mediated by the LINC complex, is intriguing and novel. Consistent with the contribution of the LINC complex to the control of developmental transitions is a recent description of differentiating keratinocytes deficient of both SUN1 and SUN2, which undergo precocious differentiation transition (Carley et al., 2021). In contrast to our results, Carley et al. demonstrated that lack of SUN proteins led to reduced repression, higher accessibility of the chromatin, and activation of terminal keratinocyte differentiation. However, this difference might stem from a distinct state of cellular differentiation of each experimental system studied. Whereas our analysis was performed on fully differentiated 
muscle fibers, the analysis of SUN-deficient keratinocytes was performed on keratinocytes prior to their terminal differentiation. Since chromatin mesoscale viscoelastic properties change during cell differentiation (Matsushita et al., 2021), the different outcome might result from the differences in chromatin properties in undifferentiated versus differentiated cells. Importantly however, the LINC complex appears to be essential for preservation of the epigenetic state of the chromatin in both cases.

A number of studies suggested a link between nuclear mechanical stimulation and chromatin epigenetic repression. For example, long-term stretch of epidermal progenitor cells led to increased $\mathrm{H} 3 \mathrm{~K} 27 \mathrm{me} 3$ occupancy resulting with silencing of epidermal differentiation (Heo et al., 2016; Le et al., 2016). Furthermore, mechanical inputs inducing nuclear deformation led to changes in the distribution of heterochromatin and euchromatin in various cell types, including cardiomyocytes and keratinocytes (Seelbinder et al., 2021; Wagh et al., 2021). Mechanistically it has been proposed that nuclear deformation changes the extent of chromatin binding to the nuclear envelope, affecting primarily repressed chromatin (Wagh et al., 2021). Interestingly, epidermal progenitor cells subjected to mechanical stretch exhibited reduced H3K9 tri-methylation associated with HP1 binding, accompanied by concomitant increase of Polycomb repressive complex 2 (PRC2) binding, resulting with enhanced global repression (Le et al., 2016). Our analysis of LINC mutant muscles, with perturbed mechanical signal transduction into the nucleus, did not reveal similar replacement between the binding of Polycomb and that of HP1, and suggests that each of these transcription repressors changed its binding independently of the other on mostly distinct set of genes, while both $\mathrm{H} 3 \mathrm{~K} 9 \mathrm{me} 3$ and $\mathrm{H} 3 \mathrm{~K} 27 \mathrm{me} 3$ repressive marks were significantly increased in the LINC mutant muscles. 
RNA-Pol II binding profile in SUN/koi mutant muscles identifies cluster 2 to contain genes with decreased binding to the transcriptional machinery complex. According to GO analysis this cluster contains a group of genes coding for muscle contractile proteins. Reduced transcription of such genes is expected to result with muscle weakening and failure in larval locomotion and indeed both phenotypes were observed in the SUN/koi mutants. Noteworthy, because the DamId analysis was performed at late stage of $3^{\text {rd }}$ instar larvae, some of the muscle-specific genes undergo transcription attenuation according to stage-specific mRNA expression data (flybase.org). For example, muscle specific genes including myosin heavy chain (MHC), sallimus (Drosophila titinlike gene), MSP300 (Nesprin-like gene), alpha actinin (Actn) and others show relative reduced mRNA levels at late $3^{\text {rd }}$ instar larval stage (Flybase). In such genes alterations of RNA-Pol II binding in the mutant muscles might not be detectable. Nevertheless, a group of other muscle genes did show a significantly reduced binding to RNA-Pol II e.g. myofilin (Mf), tropomyosinl (Tm1), myosin light chain (Mlc), actin 57B (Act57B), troponinC73F (TpnC73F) and others. In addition, a distinct group of muscle genes showed increased Polycomb binding (e.g., Zasp52, Amph, betaTub56D and others), predicting their increased repression in the SUN/koi mutant muscles. Lastly, transcription itself might be attenuated due to the increased binding of Polycomb and HP1 to components of the transcription machinery such as mediator complex proteins med10 and med28. The decreased levels of $\mathrm{H} 3 \mathrm{~K} 9$ acetylation in the $S U N / k o i$ mutant muscles is consistent with the concomitant reduction in RNA-Pol II binding to genes in cluster 2. Importantly, the lack of overlap between the group of genes that showed increased Polycomb binding (cluster 1) and the group of genes that exhibited decreased RNA Pol II binding (cluster 2) suggests that although both processes are regulated by the LINC complex, they might result from distinct effects of this complex on repressed and active chromatin regions. 
We report an increased meso-scale DNA density in the $S U N / k o i$ mutant muscles, raising the possibility that the DNA is more condensed locally in this model. This was corroborated by the DNA FISH experiment which indicated enhanced DNA compression in a region close to the centromere of chromosome 2. Similar tendency was notable in the region close to the telomere, although the difference was not statistically significant, possibly due to a greater variability obtained in the mutant nuclei. Although DNA condensation has not been addressed in LINC complex mutants in other organisms, it represents a plausible mechanism for the enhanced transcription repression. This is further supported by the specific increase in Polycomb binding we report in the LINC mutant, since chromatin compaction is the major mechanism of Polycomb mediated repression (Shao et al., 1999; Schuettengruber et al., 2017). Notably, we demonstrate that the increase in repressive $\mathrm{H} 3 \mathrm{~K} 27 \mathrm{me} 3$ and $\mathrm{H} 3 \mathrm{~K} 9 \mathrm{me} 3$ chromatin marks is driven mostly by the smaller nuclei in the SUN/koi mutant muscles, providing additional indication to the LINC mediated chromatin repression, through chromatin condensation.

So far, studies on the contribution of the LINC complex to chromatin structure have been performed in culture conditions, or in differentiating stem cells, but not in fully differentiated cells, and in live conditions. This might underlie the difference between our results indicating enhanced repression in the lack of functional LINC complex, and studies with epidermal stem cells described above. It would be important to address the contribution of the LINC complex to chromatin organization and epigenetic landscape in fully differentiated cells in other tissues, to further reveal the contribution of the LINC complex to tissue functionality.

In summary, this study analyzed the contribution of the LINC complex to chromatin epigenetics and the binding of transcriptional regulators in fully differentiated muscle fibers. We demonstrate that in the absence of functional LINC complex chromatin epigenetic repression is 
enhanced, mainly due to enhanced binding of the Polycomb transcription regulator, and a parallel decrease in the binding of RNA-Pol II, potentially through chromatin condensation-dependent mechanism. Enhanced gene repression might underly the basis for the aberrant muscle structure and function in LINC mutant muscles, as well as in cardiomyopathies and muscle dysfunction observed in LINC-associated diseases. 


\section{Materials and Methods}

Fly stocks and husbandry

The following stocks were used: koi ${ }^{84} /$ Cyo-dfd-eYfp (FBst0025105) have been described previously (Kracklauer et al., 2007), tubP-GAL80"s/TM2 (FBst0007017), GAL4-Mef2.R (FBst0027390), sls-RNAi (FBst0036108), UAS-Spastin (obtained from V. Brodu, Institute Jacques Monod, Paris), ubi-H2B-mRFP/CyO; Dr/TM6B (Bosveld et al., 2017), UAS-klar-GFP (Elhanany-Tamir et al., 2012), Tub-Gal80ts; Mef2-Gal4 (obtained from F. Schnorrer IBDM, Marseille), UAS-2E12LI-EGFP(III)/TM6B (live H3K27me3-GFP mintbody obtained from H. Kimura, Tokyo Institute of Technology). Fly lines for Targeted Dam-ID (obtained from A. Brand, The Gurdon Institute, University of Cambridge): UAS-LT3-Dm (FBtp0095492), UAS-LT3-DmRpII215 (FBtp0095495), UAS-LT3-Dam-Pc, UAS-LT3-Dam-HP1a (Marshall and Brand, 2017). All crosses were carried and maintained at $25^{\circ}, 18^{\circ}$, or $29^{\circ} \mathrm{C}$ and raised on cornmeal agar. Homozygous SUN/koi mutant larvae were selected by non-Cyo-YFP, and confirmed with mislocalization / aggregation phenotype of muscle nuclei. Temporal D-titin knockdown (sls-RNAi) and overexpression of spastin was performed using a combination of Mef2Gal4 and tubGal80ts drivers as follows: embryo collection was performed at $25^{\circ} \mathrm{C}$ for $12 \mathrm{~h}$, followed by transfer to a permissive temperature of $18^{\circ} \mathrm{C}$. Larvae were transferred to the restrictive temperature of $29^{\circ} \mathrm{C}$ for 2 days (sls-RNAi) or 1 day (spastin) before late $3^{\text {rd }}$ instar (wandering larvae). Tub-Gal80ts; Mef2Gal4 fly line was used as control for SUN/koi mutant, sls-RNAi and spastin over-expression experiments. 


\section{Targeted DamID}

We drove the expression of Dam-Polycomb, Dam-HP1 and Dam-Pol II and Dam only in control and SUN/koi mutant muscles using Mef2-gal4 driver (total 8 genotypes). Temporal control of Dam expression was achieved with a ubiquitously expressed temperature sensitive gal80. Each genotype contained 3 independent replicates, with 25 larvae per group. Eggs were laid for 6 hours and developed in $18^{\circ} \mathrm{C}$ until late larval second instar stage, and transferred to $29^{\circ} \mathrm{C}$ for 10 hours to allow muscle specific Dam expression. Larvae were then dissected and stored at $-80^{\circ} \mathrm{C}$ until all triplicates were further processed together following previously described protocol (Marshall et al., 2016). Briefly, genomic DNA was extracted and digested with methylation-specific DpnI enzymes that cleave at GATC sites. A double-stranded oligonucleotide adaptor was used to ensure directional ligation. The ligation was followed by digestion with DpnII that cuts only unmethylated GATCs. Finally, a PCR primer was used to amplify adaptor-ligated sequences. These amplified sequences were deep sequenced, the results were further analyzed by a bioinformatic pipeline (Marshall and Brand, 2015), and reads were normalized to filter out nonspecific Dam binding. Each sample was processed separately by the damid-pipeline to generate normalized ratios for each pair of samples; Dam fused to protein of interest versus Dam only (https://github.com/owenjm/damidseq_pipeline). Mean occupancy per gene was then calculated using polii script (https://github.com/owenjm/polii.gene.call). The reference genome was taken from flybase (ftp://ftp.flybase.net/releases/FB2016_02/dmel_r6.10/) and GATC sites from the damid-pipeline website. After filtering genes with DamID false discover rate (FDR) $<0.05$ (per sample), the three replicates from each group were directed to a clustering algorithm based on pairwise correlation. 
To determine the genes with statistically significant occupancy in $S U N / k o i$ mutant, relative to control, a regression on principal component analysis was performed with a z-score for each of the points around the regression line. The cut-off criteria for significantly altered binding to a gene was set to $\mathrm{FDR}<0.05$, z-score $>1.96$ (2-tailed), and GATC sites $>1$, corresponding to approximately $95 \%$ confidence interval. Independently, binding profiles (log2 fold change, normalized to Dam only) of specific genes were further visualized for control and SUN/koi mutant using the IGV browser (Robinson et al., 2011). Gene Ontology Enrichment analysis was performed with Metascape, http://metascape.org, (Zhou et al., 2019) using gene lists from the three clusters identified by K-mean clustering, with user defined background composed of all genes identified as occupied by DamID (total 7233 genes).

\section{Immunofluorescence and antibodies}

Quantitative immunofluorescence of epigenetics marks was performed on $3^{\text {rd }}$ instar, wandering larvae, that were dissected in phosphate-buffered saline (PBS) and fixed, as previously described (Wang et al., 2015). Briefly, dissected larva body walls were fixed in Paraformaldehyde (4\% from $16 \%$ stock of electron microscopy grade; Electron Microscopy Sciences, 15710) for 20 min, washed several times in PBS with 0.1\% TritonX-100, and mounted in Shandon Immu-Mount (Thermo Fisher Scientific). The following primary antibodies were used: rabbit anti-H3K9ac (Abcam, AB4441), rabbit anti-H3K9me3 (Abcam, AB176916), mouse anti-H3K27me3 (Abcam 6002). The following conjugated secondary antibodies were used: Alexa Fluor 555 goat anti-rabbit (Renium, \#A27039) and Alexa Fluor 647 goat anti- mouse (Renium, \#A21235). Hoechst 33342 (1 $\mu \mathrm{g} / \mathrm{ml}$; Sigma-Aldrich) was used for labeling DNA. 


\section{Oligopaint DNA-FISH}

Oligopaint FISH probes were designed and provided by E. Joyce, University of Pennsylvania (Rosin et al., 2018). Near centromere (488), near middle (565), and near telomere (647) probes of chromosome 2L covered 4.28Mb (18496053-22780653), 3.03Mb (9935314-12973080) and $4.4 \mathrm{Mb}$ (5273-4402412) respectively, and were designed with 42 bases of homology and densities of approximately 10 probes $/ \mathrm{kb}$.

Wandering, $3^{\text {rd }}$ instar larvae were dissected as usual and fixed for 30 minutes in RNase-free solutions. Samples were washed 3 times in 2XSSCT $(0.3 \mathrm{~m} \mathrm{NaCl}, 0.03 \mathrm{~m}$ sodium citrate, $0.1 \%$ Tween-20) at room temperature (RT), washed 10 minutes in $2 \times \mathrm{SSCT} / 50 \%$ formamide at RT, predenatured in $2 \times \mathrm{SSCT} / 50 \%$ formamide at $37^{\circ} \mathrm{C}$ for 4 hours, at $92^{\circ} \mathrm{C}$ for 3 minutes, and $60^{\circ} \mathrm{C}$ for 20 minutes. Probes were then added at $0.04 \mathrm{pmol} / \mu \mathrm{L}$ in hybridization buffer $(10 \%$ dextran sulfate/2xSSCT/50\% formamide/4\% polyvinylsulfonic acid) and samples denatured for 3 minutes at $91^{\circ} \mathrm{C}$, following by over-night incubation in $37^{\circ} \mathrm{C}$. After hybridization samples were washed twice with $2 \times \mathrm{SSCT} / 50 \%$ formamide for 30 minutes in $37^{\circ} \mathrm{C}$, washed ones $2 \times \mathrm{SSCT} / 20 \%$ formamide for 10 minutes at RT, and $4 \times 5$ minute washes $2 \times \mathrm{SSCT}$ at RT. Hoechst was added for 10 minutes $(1 \mu \mathrm{g} / \mathrm{ml})$, washed 4 times with $2 \times$ SSCT. Finally, samples were mounted on slides with ProLong Gold Antifade Mountant (Molecular probes) for imaging.

\section{Live imaging}

For imaging live nuclei in their intrinsic environment, a minimal constraint device for Drosophila larvae was designed in our laboratory, to be placed on top of a confocal microscope stage, as previously described (Lorber et al., 2020). For stationary 3D live, in-vivo imaging of muscle nuclei, selected wandering $3^{\text {rd }}$ instar larva is immersed in water for $\sim 4$ hours to decrease its 
movement (larval movement could be restored by exposure to air). For each larva, at least three nuclei were imaged from randomly chosen muscles along the entire larval body.

\section{Microscopy and Image acquisition}

Immunofluorescence images of epigenetic marks were acquired at $23^{\circ} \mathrm{C}$ on a confocal microscope Zeiss LSM 800 with a Zeiss C-Apochromat 40×/1.20 W Korr M27 lens and an Immersol W 2010 immersion medium. The samples were embedded with a coverslip high precision of $1.5 \mathrm{H} \pm 5 \mu \mathrm{m}$ (Marienfeld-Superior, Lauda-Königshofen, Germany) and acquired using Zen 2.3 software (blue edition).

Live imaging of H3K27me3-GFP was performed using an inverted Leica SP8 STED3x microscope, equipped with internal Hybrid detectors and acousto-optical tunable filter (Leica Microsystems CMS GmbH, Germany) and a white light laser excitation laser. Nuclei were imaged with a HC PL APO 86×/1.20 water STED white objective, at a scan speed of $400 \mathrm{~Hz}$, a pinhole of 0.8 A.U. and bit depth was 12 . Z-stacks were acquired with $0.308 \mu \mathrm{m}$ intervals. The acquired images were visualized during experiments using LAS-X software (Leica Application Suite X, Leica Microsystems CMS GmbH).

DNA-FISH imaging was performed using a Dragonfly spinning disk confocal system (Andor Technology PLC) connected to an inverted Leica Dmi8 microscope (Leica Microsystems CMS $\mathrm{GmbH}$ ). The signals were detected simultaneously by two sCMOS Zyla (Andor) 2048X2048 cameras, 2x2 binning, CF40 pinhole, and 12-bit depth. Images were acquired with a $63 \times / 1.3$ glycerol objective and excited with 4 different laser lines (two channels per camera): 405, 488, 561, and 637-nm laser lines. 


\section{Image Analysis}

Arivis Vision4D 3.1.2-3.4 was used for image visualization and analysis. Quantitative immunofluorescence analysis was performed with dedicated pipeline that automatically segmented multiple nuclei per stack in 3D, using denoising and Otsu threshold operation on the Hoechst channel. Nuclear volumes and total fluorescent intensities of the epigenetic marks and the DNA were calculated and exported to MATLAB R2019b (MathWorks) for further analysis. Similar algorithm of denoising and Otsu thresholding was used to segment the nuclei and the three oligopaint-FISH signals. Compartmentalization operation was further used to sort DNA FISH signals into their corresponding nuclei, and calculate distances to nuclear center.

Quantification of live H3K27me3 puncta was performed by first assessing the signal intensity profile of each nucleus and subtracting the nuclear background intensity for each nucleus individually, preserving only the bright repressive puncta. Individual puncta were then automatically segmented in 3D using Otsu operation, and puncta below volume of $0.01 \mu \mathrm{m}^{3}$ were filtered out.

\section{Statistics}

Quantitative immunofluorescence parameters from control and SUN/koi mutant nuclei were compared using a mixed linear model, with genotype as a fixed effect and with larva and muscles as random effects. The number of larvae and nuclei for each group and experiment are listed in the legends. Statistics were performed in R v.4.0.2 using the package lmerTest v.3.1-2. Linear fits to the epigenetic mark intensities, and total chromatin as function of the nuclear volume were generated using linear mixed-effects model fit. BoxPlotR was used to generate box plots (Spitzer et al., 2014), in which the center lines represent the medians, box limits indicate 25th and 75 th 
percentiles, and whiskers, determined by using the Tukey method, extended to data points $<1.5$ interquartile ranges from the first and third quartiles as determined by the BoxPlotR software.

\section{Acknowledgments}

We thank Andrea Brand (Gordon Institute, University of Cambridge, England), Hiroshi Kimura (Tokyo Inst. of Technology, Tokyo, Japan), and Bloomington Stock Center for providing essential fly lines. We also thank Developmental Studies Hybridoma Bank (DSHB) for antibodies, and FlyBase for important genomic data. We are grateful for Eric Joyce (University of Pennsylvania, US) for providing us with the oligopaint probes and protocols. We thank the Nancy\&Stephen Grand Israel National Center for Personalized Medicine (G-INCPM), and especially Eviatar Weizman, for processing and analyzing the DamId experiments. We thank R. Rotkopf from the Life Sciences Core Facility, Weizmann Institute, for statistical analysis. The images in this paper were acquired at the Advanced Optical Imaging Unit, de Picciotto-Lesser Cell Observatory unit, at the Moross Integrated Cancer Center Life Science Core Facilities, Weizmann Institute of Science. This study was supported by grants from "The French Muscular Dystrophy Association (AFM-Téléthon)" grant \# 22339 and Israel Science Foundation (ISF) grant \# 750/17. 


\section{Supplementary Information}

A

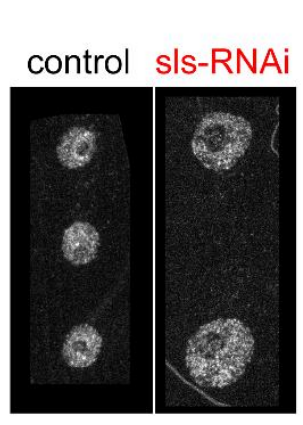

$A^{\prime}$

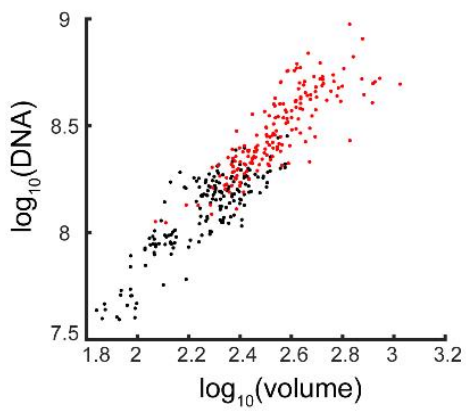

B

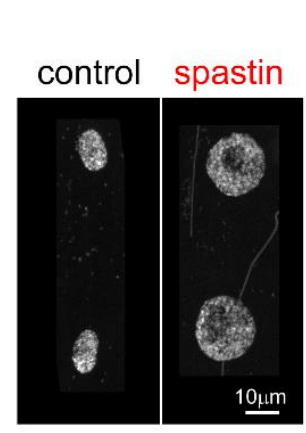

B'

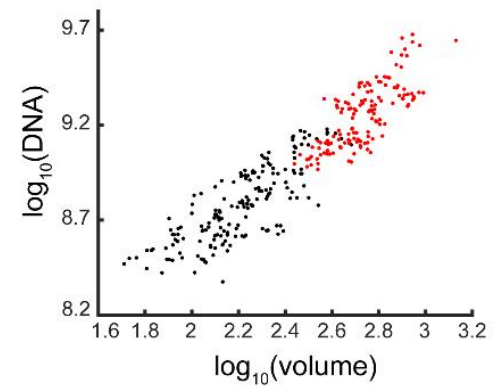

Figure S1: Perturbed forces from the sarcomeres or microtubule network increase nuclei size, with scaled increase in DNA content, in Drosophila $3^{\text {rd }}$ instar larva muscles. (A) Muscle nuclei labeled with Hoechst for control, and muscle specific knockdown of D-titin (sls-RNAi) for 2 days. (A') Total DNA scales linearly with nuclear volume ( $\log 10$ scale) for control (black dots) and sls-RNAi (red dots), despite larger nuclei in the latter group. $\mathrm{N}=5$ larvae, $\mathrm{n}=170$ in control, $\mathrm{N}=5$ larvae, $\mathrm{n}=177$ nuclei in $s l s$-RNAi (B and B') Similar analysis for control and muscle specific overexpression of spastin for 1 day. $\mathrm{N}=5$ larvae, $\mathrm{n}=174$ in control, $\mathrm{N}=5$ larvae, $\mathrm{n}=140$ nuclei in spastin. 


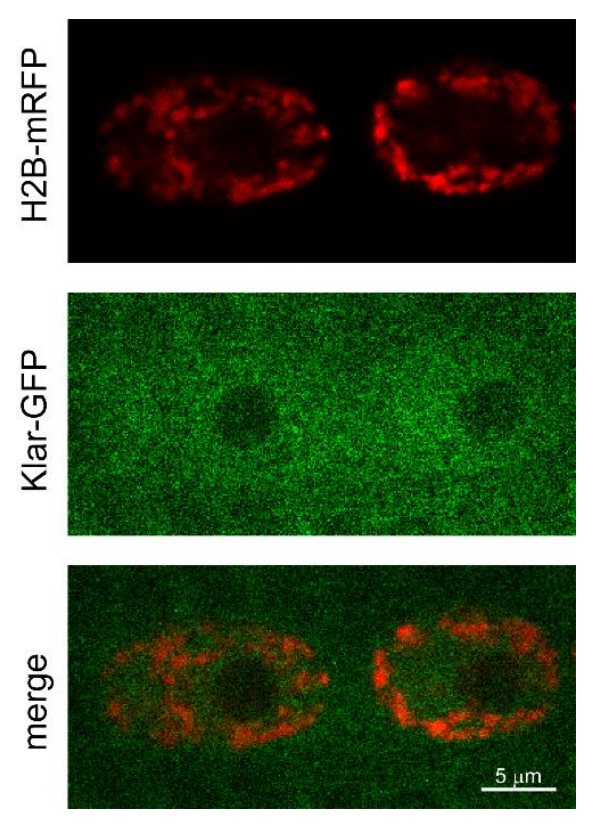

Figure S2: Live imaging of chromatin distribution in SUN/koi mutant muscle nuclei. Middle confocal section of muscle nuclei co-labeled with H2B-mRFP, and Klar-GFP. Peripheral chromatin distribution in the $S U N / k o i$ mutant can be observed from the H2B-mRFP channel. KlarGFP fails to localize to the nuclear envelope in the $S U N / k o i$ mutant.

\section{Movie S1: 3D spatial distribution of H3K27me3-GFP in live, in-vivo control muscle} nucleus. 3D reconstruction from confocal z-stacks acquired with $0.308 \mu \mathrm{m}$ intervals.

Movie S2: 3D spatial distribution of H3K27me3-GFP in live, in-vivo SUN/koi mutated muscle nuclei. 3D reconstruction from confocal z-stacks acquired with $0.308 \mu \mathrm{m}$ intervals. 


\section{References}

Amiad-Pavlov, D., D. Lorber, G. Bajpai, A. Reuveny, F. Roncato, R. Alon, S. Safran, and T. Volk. 2021. Live imaging of chromatin distribution reveals novel principles of nuclear architecture and chromatin compartmentalization. Sci. Adv. 7:eabf6251. doi:10.1126/sciadv.abf6251.

Bosveld, F., A. Ainslie, and Y. Bellaïche. 2017. Sequential activities of Dynein, Mud and Asp in centrosome-spindle coupling maintain centrosome number upon mitosis. J. Cell Sci. 130:3557-3567. doi:10.1242/jcs.201350.

Carley, E., R.K. Stewart, A. Zieman, I. Jalilian, D.E. King, A. Zubek, S. Lin, V. Horsley, and M.C. King. 2021. The linc complex transmits integrin-dependent tension to the nuclear lamina and represses epidermal differentiation. Elife. 10. doi:10.7554/eLife.58541.

Cartwright, S., and I. Karakesisoglou. 2014. Nesprins in health and disease. Semin. Cell Dev. Biol. 29:169-179. doi:10.1016/j.semcdb.2013.12.010.

Chang, W., H.J. Worman, and G.G. Gundersen. 2015. Accessorizing and anchoring the LINC complex for multifunctionality. J. Cell Biol. 208:11-22. doi:10.1083/JCB.201409047.

Elhanany-Tamir, H., Y. V. Yu, M. Shnayder, A. Jain, M. Welte, and T. Volk. 2012. Organelle positioning in muscles requires cooperation between two KASH proteins and microtubules. J. Cell Biol. 198:833-846. doi:10.1083/jcb.201204102.

Filion, G.J., J.G. van Bemmel, U. Braunschweig, W. Talhout, J. Kind, L.D. Ward, W. Brugman, I.J. de Castro, R.M. Kerkhoven, H.J. Bussemaker, and B. van Steensel. 2010. Systematic Protein Location Mapping Reveals Five Principal Chromatin Types in Drosophila Cells. Cell. 143:212-224. doi:10.1016/J.CELL.2010.09.009.

Ghosh, S., J.M. Gardner, C.J. Smoyer, J.M. Friederichs, J.R. Unruh, B.D. Slaughter, R. Alexander, R.D. Chisholm, K.K. Lee, J.L. Workman, and S.L. Jaspersen. 2012. Acetylation of the SUN protein Mps3 by Eco1 regulates its function in nuclear organization. Mol. Biol. Cell. 23:2546-2559. doi:10.1091/MBC.E11-070600/ASSET/IMAGES/LARGE/2546FIG8.JPEG.

Heffler, J., P.P. Shah, P. Robison, S. Phyo, K. Veliz, K. Uchida, A. Bogush, J. Rhoades, R. Jain, and B.L. Prosser. 2019. A Balance Between Intermediate Filaments and Microtubules Maintains Nuclear Architecture in the Cardiomyocyte. Circ. Res. CIRCRESAHA.119.315582. doi:10.1161/CIRCRESAHA.119.315582.

Heo, S.-J., W.M. Han, S.E. Szczesny, B.D. Cosgrove, D.M. Elliott, D.A. Lee, R.L. Duncan, and R.L. Mauck. 2016. Mechanically Induced Chromatin Condensation Requires Cellular Contractility in Mesenchymal Stem Cells. Biophys. J. 111:864-874. doi:10.1016/J.BPJ.2016.07.006.

Hernandez, M., J. Patzig, S.R. Mayoral, K.D. Costa, J.R. Chan, and P. Casaccia. 2016. Mechanostimulation Promotes Nuclear and Epigenetic Changes in Oligodendrocytes. $J$. Neurosci. 36:806-813. doi:10.1523/JNEUROSCI.2873-15.2016.

Horn, H.F. 2014. LINC Complex Proteins in Development and Disease. Curr. Top. Dev. Biol. 109:287-321. doi:10.1016/B978-0-12-397920-9.00004-4.

Kirby, T.J., and J. Lammerding. 2018. Emerging views of the nucleus as a cellular mechanosensor. Nat. Cell Biol. 20:373-381. doi:10.1038/s41556-018-0038-y.

Kracklauer, M.P., S.M.L. Banks, X. Xie, Y. Wu, and J.A. Fischer. 2007. Drosophila klaroid Encodes a SUN Domain Protein Required for Klarsicht Localization to the Nuclear Envelope and Nuclear Migration in the Eye. http://dx.doi.org/10.4161/fly.4254. 1:75-85. 
doi:10.4161/FLY.4254.

Le, H.Q., S. Ghatak, C.-Y.C. Yeung, F. Tellkamp, C. Günschmann, C. Dieterich, A. Yeroslaviz, B. Habermann, A. Pombo, C.M. Niessen, and S.A. Wickström. 2016. Mechanical regulation of transcription controls Polycomb-mediated gene silencing during lineage commitment. Nat. Cell Biol. 18:864-875. doi:10.1038/ncb3387.

Lityagina, O., and G. Dobreva. 2021. The LINC Between Mechanical Forces and Chromatin. Front. Physiol. 12:1242. doi:10.3389/FPHYS.2021.710809/BIBTEX.

Lorber, D., R. Rotkopf, and T. Volk. 2020. A minimal constraint device for imaging nuclei in live Drosophila contractile larval muscles reveals novel nuclear mechanical dynamics. Lab Chip. doi:10.1039/D0LC00214C.

Marshall, O.J., and A.H. Brand. 2015. damidseq_pipeline: an automated pipeline for processing DamID sequencing datasets. Bioinformatics. 31:3371-3373. doi:10.1093/bioinformatics/btv386.

Marshall, O.J., and A.H. Brand. 2017. Chromatin state changes during neural development revealed by in vivo cell-type specific profiling. Nat. Commun. 8:2271. doi:10.1038/s41467017-02385-4.

Marshall, O.J., T.D. Southall, S.W. Cheetham, and A.H. Brand. 2016. Cell-type-specific profiling of protein-DNA interactions without cell isolation using targeted DamID with next-generation sequencing. Nat. Protoc. 2016 119. 11:1586-1598. doi:10.1038/nprot.2016.084.

Matsushita, K., C. Nakahara, S. Kimura, N. Sakamoto, S. Ii, and H. Miyoshi. 2021. Intranuclear mesoscale viscoelastic changes during osteoblastic differentiation of human mesenchymal stem cells. FASEB J. 35:e22071. doi:10.1096/FJ.202100536RR.

Méjat, A., and T. Misteli. 2010. LINC complexes in health and disease. http://dx.doi.org/10.4161/nucl.1.1.10530. 1:40-52. doi:10.4161/NUCL.1.1.10530.

Poulet, A., C. Duc, M. Voisin, S. Desset, S. Tutois, E. Vanrobays, M. Benoit, D.E. Evans, A. V. Probst, and C. Tatout. 2017. The LINC complex contributes to heterochromatin organisation and transcriptional gene silencing in plants. J. Cell Sci. 130:590-601. doi:10.1242/JCS.194712/265211/AM/THE-LINC-COMPLEX-CONTRIBUTES-TOHETEROCHROMATIN.

Puckelwartz, M.J., E. Kessler, Y. Zhang, D. Hodzic, K.N. Randles, G. Morris, J.U. Earley, M. Hadhazy, J.M. Holaska, S.K. Mewborn, P. Pytel, and E.M. McNally. 2009. Disruption of nesprin-1 produces an Emery Dreifuss muscular dystrophy-like phenotype in mice. Hum. Mol. Genet. 18:607-620. doi:10.1093/HMG/DDN386.

Razafsky, D., and D. Hodzic. 2009. Bringing KASH under the SUN: the many faces of nucleocytoskeletal connections. J. Cell Biol. 186:461-472. doi:10.1083/JCB.200906068.

Robinson, J.T., H. Thorvaldsdóttir, W. Winckler, M. Guttman, E.S. Lander, G. Getz, and J.P. Mesirov. 2011. Integrative genomics viewer.

Rosin, L.F., S.C. Nguyen, and E.F. Joyce. 2018. Condensin II drives large-scale folding and spatial partitioning of interphase chromosomes in Drosophila nuclei. PLoS Genet. 14:e1007393. doi:10.1371/journal.pgen.1007393.

Schuettengruber, B., H.M. Bourbon, L. Di Croce, and G. Cavalli. 2017. Genome Regulation by Polycomb and Trithorax: 70 Years and Counting. Cell. 171:34-57. doi:10.1016/J.CELL.2017.08.002.

Seelbinder, B., S. Ghosh, S.E. Schneider, A.K. Scott, A.G. Berman, C.J. Goergen, K.B. Margulies, K.C. Bedi, E. Casas, A.R. Swearingen, J. Brumbaugh, S. Calve, and C.P. Neu. 
2021. Nuclear deformation guides chromatin reorganization in cardiac development and disease. Nat. Biomed. Eng. 2021. 1-17. doi:10.1038/s41551-021-00823-9.

Shao, Z., F. Raible, R. Mollaaghababa, J.R. Guyon, C.T. Wu, W. Bender, and R.E. Kingston. 1999. Stabilization of Chromatin Structure by PRC1, a Polycomb Complex. Cell. 98:37-46. doi:10.1016/S0092-8674(00)80604-2.

Sosa, B.A., A. Rothballer, U. Kutay, and T.U. Schwartz. 2012. LINC Complexes Form by Binding of Three KASH Peptides to Domain Interfaces of Trimeric SUN Proteins. Cell. 149:1035-1047. doi:10.1016/J.CELL.2012.03.046.

Southall, T.D., K.S. Gold, B. Egger, C.M. Davidson, E.E. Caygill, O.J. Marshall, and A.H. Brand. 2013. Cell-Type-Specific Profiling of Gene Expression and Chromatin Binding without Cell Isolation: Assaying RNA Pol II Occupancy in Neural Stem Cells. Dev. Cell. 26:101-112. doi:10.1016/j.devcel.2013.05.020.

Spitzer, M., J. Wildenhain, J. Rappsilber, and M. Tyers. 2014. BoxPlotR: a web tool for generation of box plots. Nat. Methods 2014 112. 11:121-122. doi:10.1038/nmeth.2811.

Tapley, E.C., and D.A. Starr. 2013. Connecting the nucleus to the cytoskeleton by SUN-KASH bridges across the nuclear envelope. Curr. Opin. Cell Biol. 25:57-62.

doi:10.1016/J.CEB.2012.10.014.

Tatavosian, R., S. Kent, K. Brown, T. Yao, H.N. Duc, T.N. Huynh, C.Y. Zhen, B. Ma, H. Wang, and X. Ren. 2019. Nuclear condensates of the Polycomb protein chromobox 2 (CBX2) assemble through phase separation. J. Biol. Chem. 294:1451-1463. doi:10.1074/JBC.RA118.006620.

Titlow, J., F. Robertson, A. Järvelin, D. Ish-Horowicz, C. Smith, E. Gratton, and I. Davis. 2020. Syncrip/hnRNP Q is required for activity-induced Msp300/Nesprin-1 expression and new synapse formation. J. Cell Biol. 219. doi:10.1083/JCB.201903135/133707.

Tjalsma, S.J.D., M. Hori, Y. Sato, A. Bousard, A. Ohi, A.C. Raposo, J. Roensch, A. Le Saux, J. Nogami, K. Maehara, T. Kujirai, T. Handa, S. Bagés-Arnal, Y. Ohkawa, H. Kurumizaka, S.T. da Rocha, J.J. Żylicz, H. Kimura, and E. Heard. 2021. H4K20me1 and H3K27me3 are concurrently loaded onto the inactive $\mathrm{X}$ chromosome but dispensable for inducing gene silencing. EMBO Rep. 22:e51989. doi:10.15252/EMBR.202051989.

Wagh, K., M. Ishikawa, D.A. Garcia, D.A. Stavreva, A. Upadhyaya, and G.L. Hager. 2021. Mechanical Regulation of Transcription: Recent Advances. Trends Cell Biol. 31:457-472. doi:10.1016/J.TCB.2021.02.008.

Wallrath, L.L., J. Bohnekamp, and T.M. Magin. 2016. Cross talk between the cytoplasm and nucleus during development and disease. Curr. Opin. Genet. Dev. 37:129-136. doi:10.1016/J.GDE.2016.03.007.

Wang, S., A. Reuveny, and T. Volk. 2015. Nesprin provides elastic properties to muscle nuclei by cooperating with spectraplakin and EB1. J. Cell Biol. 209:529-538. doi:10.1083/jcb.201408098.

Wang, S., E. Stoops, U. CP, B. Markus, A. Reuveny, E. Ordan, and T. Volk. 2018. Mechanotransduction via the LINC complex regulates DNA replication in myonuclei. $J$. Cell Biol. 217:2005-2018. doi:10.1083/jcb.201708137.

De Wit, E., F. Greil, and B. Van Steensel. 2007. High-Resolution Mapping Reveals Links of HP1 with Active and Inactive Chromatin Components. PLOS Genet. 3:e38. doi:10.1371/JOURNAL.PGEN.0030038.

Zhou, Y., B. Zhou, L. Pache, M. Chang, A.H. Khodabakhshi, O. Tanaseichuk, C. Benner, and S.K. Chanda. 2019. Metascape provides a biologist-oriented resource for the analysis of 
bioRxiv preprint doi: https://doi.org/10.1101/2022.02.09.479725; this version posted February 10, 2022. The copyright holder for this preprint (which was not certified by peer review) is the author/funder, who has granted bioRxiv a license to display the preprint in perpetuity. It is made available under aCC-BY-NC-ND 4.0 International license.

systems-level datasets. Nat. Commun. 10. doi:10.1038/S41467-019-09234-6. 